A puntes del CENES

ISSN 0120-3053

Vol. XXIX - №. 50

Págs. $151-171$

II Semestre de 2010

\title{
Los dos gobiernos de Alfonso López Pumarejo: estado y reformas económicas y sociales en Colombia (1934-1938, 1942-1945)
}

Óliver M ora Toscano*

Fecha de recepción: 18 de agosto de 2010

F echa de aprobación: 30 de septiembre de 2010

Clasificación JEL: H12, H50, N 10, E60, E61

Docente asistente de tiempo completo, Escuela de Economía, Universidad Pedagógica y Tecnológica de Colombia, UPTC. Actualmente en comisión de estudios de Doctorado en Economía con énfasis en Economía del Desarrollo en la Universidad Federal del Río Grande do Sul (UFRGS), Porto Alegre, Brasil. Correo electrónico: olivermora99@yahoo.es 


\section{Resumen}

Este artículo analiza brevemente la intencionalidad reformadora de uno de los gobiernos más importantes en Colombia durante el siglo XX, el de Alfonso López Pumarejo. Con este propósito se consideran, específicamente, los principales antecedentes, el contexto general de los dos gobiernos comprendidos entre 1934 a 1938 y 1942 a 1945, las reformas económicas y sociales intentadas y, finalmente, una breve interpretación de dichas reformas en el contexto de ese momento histórico.

Palabras clave: estado, crisis económica, reformas económicas y sociales, modelo de acumulación, política económica, intencionalidad reformadora.

\section{Abstract}

This article briefly reviews the reformist intentions of one of the major governments in Colombia during the tw entieth century, that of Alfonso Lopez. For this purpose, consider specifically, the main background, the general context of the two governments included from 1934 to 1938 and 1942 to 1945, economic and social reforms attempted to carry out and, finally, a brief interpretation of these reforms in the context of this historic moment.

Key words: state, economic crisis, economic and social reforms, a model of accumulation, economic policy, intent to reform. 


\section{Introducción}

La gestión de gobierno del estadista colombiano A Ifonso López Pumarejo es considerada por varios analistas como una de las más destacadas e importantes del siglo XX en Colombia. El conjunto de políticas y reformas ejecutadas durante sus administraciones abrieron el camino para grandes transformaciones en el papel del Estado, la economía, la industria, la tenencia de la tierra, la educación pública, las relaciones laborales, entre otros aspectos políticos, económicos y sociales.

Alfonso López Pumarejo gobernó Colombia durante dos períodos. EI primero, entre 1934 y 1938, y el segundo, entre 1942 y 1945. De los dos períodos, el más relevante por el conjunto de transformaciones propuestas y realizadas es el primero. Durante su segundo período enfrentó grandes limitaciones que le impidieron continuar a profundidad su labor reformista.

Este artículo tiene como objetivo efectuar un breve análisis de las reformas económicas y sociales propuestas y ejecutadas por el presidente López entre 1934 a 1938 y 1942 a 1945. El propósito principal de las reformas era "modernizar" las relaciones económicas y sociales existentes hasta ese momento en Colombia, las cuales respondían en buena parte a las características del siglo $\mathrm{XIX}$.

El eje central de las transformaciones propuestas es el cambio en el concepto y el papel del Estado colombiano en la economía y la sociedad. L a importancia de tales transformaciones no se mide tanto por los resultados cuantitativos como tal, sino sobre todo por la 
"intencionalidad" de mudanza que de alguna u otra manera constituyeron un precedente de gran importancia hacia el futuro.

A partir de los anteriores elementos, en este documento se presentan, en primer lugar, los antecedentes. A continuación, se esboza el contexto general de los dos períodos de gobierno. Posteriormente, se anal iza la intencionalidad reformadora de Alfonso López Pumarejo. Esta intencionalidad se expresa, en general, en el conjunto de reformas llevadas a cabo en Colombia entre 1934 a 1938 y 1942 a 1945. Finalmente, en esta artículo se elabora una breve interpretación de las reformas económicas y sociales implementadas, esto a manera de conclusión.

\section{Antecedentes}

En 1930 el partido Liberal vuelve al poder después de cuatro décadas de dominación política por parte de los conservadores, con excepción del período 1909-1914. El presidente liberal elegido en ese año fue Enrique Olaya Herrera. Sin embargo, dado que se trataba de una etapa de transición, su gobierno resultó ser realmente una coalición entre liberal es y conservadores opuestos a la dirigencia oficial de este último sector político, específicamente opuestos al dirigente histórico del conservatismo L aureano Gómez. En virtud a esa característica, este gobierno liberal de coalición fue denominado por el presidente Olaya como de "concentración nacional" ${ }^{2}$.

Olaya Herrera heredó de las administraciones conservadoras que lo precedieron un conjunto importante de obras públicas, de infraestructura de servicios básicos y de comunicaciones. Estas obras fueron financiadas en una proporción importante con el pago hecho, a mediados de la década de los veinte, por Estados Unidos al gobierno colombiano como indemnización por la pérdida de Panamás.

N o obstante, la crítica principal que los liberales esgrimían contra el régimen conservador era en relación con el atraso social en que se encontraba el país respecto, no ya de las naciones avanzadas del mundo, sino de otras de A mérica latina, lo cual dejaba a Colombia en un lugar muy rezagado del escenario latinoamericano. Por ello, a partir de 1921 se estableció la promoción de reformas sociales incluyentes como una de las metas más importantes del liberalismo ${ }^{4}$.

El gobierno de Olaya Herrera inaugura una etapa muy importante en la historia

MOLINA, Gerardo, (2007), Las ideas liberales en Colombia: 1849-1959, 5. edición, Universidad Libre, 20, Bogotá.

3 ROBINSON, James y URRUTIA, Miguel, (2007), Economía colombiana del siglo XX. Un análisis cuantitativo, Fondo de Cultura Económica - Banco de la República, 393, Bogotá.

4 SANTOS, Enrique, (2005), “Las revolucionaria República Liberal”, en Revista Credencial Historia, N.o. 183, 1, Bogotá. 
económica y política de Colombia, la denominada "segunda república Liberal". La misma cubre el período 1930 - 1946, intervalo de tiempo durante el cual se sucedieron cuatro gobiernos consecutivos: Enrique Olaya Herrera entre 1930 y 1934, A Ifonso López Pumarejo (primer gobierno) entre 1934 y 1938, Eduardo Santos entre 1938 y 1942, nuevamente Alfonso López Pumarejo (segundo gobierno) entre 1942 y 1945 y, finalmente, Alberto Lleras Camargo (quien completa el período formal de López ante la renuncia del mismo en 1945) entre 1945 y 1946.

De todos estos gobiernos, el más destacado, por tratar de introducir un conjunto de reformas económicas y sociales adelantadas para su época, fue el primer período de Alfonso López Pumarejo (1934-1938), gobierno que marcó una etapa muy importante en la historia de Colombia 5 . Esa importancia, más que por sus resultados, se da por las "intencionalidad" de llevar a cabo unas reformas que de alguna u otra manera constituyeron un precedente de gran importancia hacia el futuro.

\section{L os dos gobiernos de Alfonso L ópez Pumarejo. Contexto general}

A continuación se presentan los aspectos generales más importantes de los dos gobiernos de A Ifonso López. La primera administración comprende el período 1934 a 1938; entre tanto, el segundo gobierno tiene lugar entre 1942 a 1945. Como ya se mencionó, el período formal de este segundo turno es hasta 1946, pero López renuncia un año antes de su culminación.

\subsection{Primera administración: "L a revolución en marcha" (1934-1938)}

Para las elecciones de 1934, el partido conservador se abstuvo de participar en las mismas, ante la evidente mayor popularidad del Partido Liberal y la perspectiva entonces de una posible derrota de importante magnitud. En este contexto favorable, el candidato del Partido Liberal, Alfonso López Pumarejo, lograría el triunfo con un número importante de votos, 938.000, la mayor votación registrada hasta entonces en la historia del país $s^{6}$. En dichas elecciones, López sólo tuvo como competidor al candidato del Partido Comunista.

El partido liberal colombiano, ganador por segunda vez consecutiva, se sentía identificado, en cierto sentido, con las corrientes renovadoras que habían triunfado en otras regiones del mundo como la revolución mexicana, la república española e inclusive el "N ew

5 TIRADO, Álvaro (compilador), (1986), Estado y economía: 50 años de la reforma del 36, Contraloría General de la República, 16, Bogotá.

6 URREGO, Miguel, (2005), La revolución en marcha en Colombia (1934-1938): Una lectura en perspectiva latinoamericana, Universidad Michoacana de San Nicolás de Hidalgo - Instituto de Investigaciones Históricas, 65, Michoacán, México. 
Deal" de Franklin D. Roosevelt ${ }^{7}$. La Constitución de 1886 aparecía entonces como una carta teocrática, apta para una república rural, atrasada y tradicionalista.

L a crisis o gran depresión de 1929, junto con los graves conflictos rurales y obreros de la década del veinte, parecían exigir nuevos instrumentos económicos y legales. Esta crisis generó una disminución brusca de los flujos de financiamiento externo provenientes principalmente de los Estados Unidos, con efectos negativos sobre el crecimiento y el empleo ${ }^{8}$. A este contexto adverso, se le une también la caída de los precios del café a finales de 1928. El liberalismo planteaba, como respuesta a estos desafíos, un programa de Estado intervencionista, capaz de orientar la vida económica y de colocarse como árbitro en los conflictos entre las distintas clases sociales ${ }^{10}$.

El gobierno de Alfonso López, elegido en 1934, asumió de forma inmediata el propósito de reformar la carta constitucional, y desde septiembre presentó al Congreso diversos proyectos en esta dirección. Él mismo bautizó su primer gobierno como el de la " $\mathrm{La}$ Revolución en M archa".
El proceso vivido entre 1934 y 1938 en Colombia no fue una revolución en el sentido literal de la palabra, y tal como lo indicaba el nombre dado al mismo. En efecto, no era éste el objetivo principal que buscaban sus principales promotores, Alfonso López Pumarejo, A lberto L leras Camargo y Darío Echandía. Sin embargo, sí representó para las condiciones de la época, e inclusive desde una perspectiva de largo plazo, un esfuerzo importante de iniciar un conjunto de transformaciones en un país cuyas estructuras todavía se encontraban en parte anquilosadas en el conservadurismo y el atraso económico y social de finales del siglo XIX ${ }^{11}$. Se buscaba así adecuar la concepción del liberalismo vigente en ese momento a las condiciones de un país en desarrollo y con grandes limitaciones de carácter estructural.

López Pumarejo pertenecía a la aristocracia financiera, y como miembro "ilustrado" de la misma, conocía la evolución del pensamiento occidental del momento. A su alrededor aglutinó a la intelectualidad del partido Liberal, una intelectualidad que conocía las transformaciones del mundo, la lucha de las democracias occidentales contra los regímenes autoritarios y fascistas ${ }^{12}$. Ese

MELO, Jorge Orlando, (1991), "Las reformas liberales de 1936 y 1968. Progreso social y reorganización del Estado", en Revista Credencial Historia, N.o 13, 1, Bogotá.

8 OCAMPO, José Antonio y MONTENEGRO, Santiago, (2007), Crisis mundial, protección e industrialización. Editorial Norma, 2. ${ }^{\text {a }}$ edición, 20, Bogotá.

9 lbid, 23.

10 KALMANÓVITZ, Salomón, (2003), Economía y nación: Una breve historia de Colombia, 2.․․ edición, Editorial Norma, 366, Bogotá.

11 TIRADO, Álvaro, (1995), Aspectos políticos del primer gobierno de Alfonso López Pumarejo, Editorial Planeta, 35, Bogotá.

12 GIRALDO, César, (1994), Estado y hacienda pública en Colombia 1934-1990, Contraloría General de la República, 14 15, Bogotá. 
pensamiento autoritario era defendido por la gran mayoría de dirigentes del partido Conservador, quienes representaban principalmente los intereses económicos de los grandes propietarios de la tierra ${ }^{13}$.

En su concepción, el Estado colombiano debía ser reformado para que creara las condiciones económicas y sociales necesarias para viabilizar un nuevo proceso de acumulación basado en el mercado interno. $Y$ ese Estado debía ser intervencionista, defensor de la industria y la agricultura frente a la competencia externa y benefactor de los sectores populares, introduciendo reformas en la legislación social y en la tenencia de la tierra. Esto permitiría ampliar la capacidad de consumo de la población en general y crear así nuevos mercados y nuevas fuentes de acumulación, tal como lo expresó el mismo López en su mensaje al congreso en $1935^{14}$.

En general, una parte importante de la élite social y económica del momento, principalmente los grandes terratenientes propietarios de la tierra, manifestó una fuerte oposición al conjunto de cambios propuestos por el gobierno durante su primera administración ${ }^{15}$. Se de sectores muy importantes de la oligarquía urbana y rural que observaba cómo sus intereses económicos podían ser afectados con las propuestas reformistas del nuevo gobierno.

Esta oposición se organizó principalmente alrededor del Partido Conservador. M ás adelante, también fue creciente la defensa de estos intereses dentro del mismo partido de gobierno, el Partido Liberal. Esto propició la práctica división del liberalismo entre los defensores del gobierno y aquellos que propendían por cambios más moderados. Algunos acusaron a López de ser comunista y los conservadores adelantaron una campaña de oposición que se basaba en luchar contra la influencia comunista en el Gobierno. Este contexto limitó de una forma importante el alcance de las transformaciones propuestas por el gobierno.

López no se amedrentó ante esta férrea oposición de la oligarquía tanto liberal como conservadora y, por el contrario, la desafió. A diferencia de Olaya Herrera, prescindió de un gobierno bi partidista de coalición e integró el suyo con mayoría casi absoluta de liberales, y unos pocos conservadores.

L as propuestas presentadas por el gobierno incluían una nueva concepción del Estado como regulador de la vida económica y social, nuevas formulaciones del derecho de propiedad, un

\footnotetext{
13 Ibid, 15.

14 LÓPEZ PUMAREJO, Alfonso, (1979), Obras selectas, dos(2) volúmenes, compilación de Jorge Mario Eastman, Imprenta Nacional - Cámara de Representantes de Colombia, 20, Bogotá.

15 KALMANÓVITZ, Salomón, (2003), Op. cit., 365.
} 
replanteamiento de las relaciones entre el Estado y la Iglesia, y una serie de medidas modernizadoras en el campo social y político ${ }^{16}$. Esto reflejaba en buena parte su pensamiento brevemente resumido atrás.

Los propósitos de cambio se expresaron en un conjunto de reformas en los ámbitos constitucional, agrario, tributario, judicial, educativo, laboral y de política internacional. Estas reformas produjeron en Colombia una transformación fundamental en la concepción que se tenía hasta el momento respecto del papel del Estado en la economía y en la sociedad.

\section{2 Segunda administración (1942-1945)}

Eduardo Santos fue el sucesor de López durante el período constitucional 19381942. Santos pertenecía al ala moderada del liberalismo, la cual era más partidaria de dar una pausa al ritmo de reformas impulsadas por López Pumarejo, sobre todo a comienzos de su primera administración. Durante el gobierno de Santos no se establecieron nuevas leyes reformistas y no se aplicaron a profundidad las reformas ya aprobadas entre 1934 y 1938.

Tras finalizar su mandato en 1938, Alfonso López viaja al exterior y regresa a Colombia a principios de 1942 con la intención de volver a gobernar. EI sector moderado del liberalismo, encabezado por el presidente saliente, Eduardo Santos, no respaldó a López, postulando al destacado ex ministro Carlos L ozano en la convención liberal, pese a lo cual López ganó la candidatura oficial del Partido. En ese escenario, el sector moderado del Liberalismo decide no aceptar la candidatura única de L ópez y proponen entonces un candidato disidente, resultando finalmente escogido Carlos A rango V élez a nombre de esa disidencia Liberal.

A Irededor de A rango se agruparon los sectores políticos y económicos que estaban en contra de una segunda etapa de reformas en cabeza de López Pumarejo. Importantes grupos económicos y los grandes propietarios rurales se unieron a esta candidatura disidente. El Partido Conservador, encabezado por Laureano Gómez, se consideró incapaz de ganar postulando un candidato propio, por lo que ofreció el respaldo al candidato de la disidencia liberal, es decir, apoyaron al candidato Carlos A rango.

Se trató de una elección más reñida que la de 1934. López logra vencer todos estos obstáculos y resulta finalmente vencedor, siendo elegido entonces presidente para el período 1942-1946.

D urante su segundo mandato, que inició el 7 de agosto de 1942, López no consiguió reunir el apoyo político

$\overline{16}$ MELO, Jorge Orlando, (1991), Op. cit., 1. 
suficiente para sacar adelante nuevas reformas y, por el contrario, se enfrentó a un panorama de muy dura oposición. Ésta se encontraba encabezada por buena parte de la élite económica del país. Políticamente esta situación adversa se manifiesta en la oposición del Partido Conservador y de una parte importante del mismo Partido de gobierno, específicamente, los sectores moderados y de derecha del liberalismo.

La difícil situación económica ocasionada por la segunda guerra mundial y por la baja de los precios del café impidió a López adelantar su programa de reformas sociales, junto con la paulatina disminución de los márgenes de gobernabilidad ante las profundas críticas provenientes de los sectores opositores ${ }^{17}$. La situación política se debilitó tanto que en 1944 un grupo de militares intentó dar un golpe de Estado pero fracasó debido a divisiones internas dentro de las mismas fuerzas armadas y al apoyo popular que recibió el gobierno a través de grandes manifestaciones a su favor. A nte este contexto, y por otras circunstancias de tipo personal, en 1945 López renuncia de manera irrevocable ante el Congreso Nacional. Su M inistro de Relaciones Exteriores y designado presidencial, A I berto L leras Camargo, completará el período presidencial formal hasta 1946 , año en el cual los conservadores vuelven nuevamente al poder político del Estado.
A pesar de este ambiente en contra y el contexto claramente desfavorable a su gestión, López Pumarejo logra implementar dos importantes reformas en este período, la reforma constitucional de 1945 y la reforma laboral. Los fundamentos conceptuales de esta última fueron construidos durante su primera administración.

4. La intencionalidad reformadora: L as reformas económicas y sociales realizadas durante las dos administraciones de Alfonso López Pumarejo

La intencionalidad reformadora de A Ifonso López se expresa en el conjunto de reformas económicas y sociales realizadas durante sus dos períodos de gobierno, en especial durante el primero comprendido entre 1934 y 1938. Estas reformas son, principalmente, la reforma constitucional de 1936, la reforma agraria, la reforma educativa, la reforma tributaria, la reforma laboral y final mente la reforma constitucional de 1945.

\section{4. $1 \mathrm{~L}$ a reforma constitucional de 1936}

La reforma constitucional de 1936 cambió la concepción del Estado hasta ese momento vigente en Colombia. M ediante la misma se transforma el concepto del Estado gendarme, propia de la Constitución Nacional de 1886, por la del Estado como una entidad con una

17 Ibid, 161. 
mayor capacidad de intervención en la economía y, en general, en la sociedad ${ }^{18}$. Esta reforma tuvo influencias de la Constitución de la Segunda República Española de 1931 y, en ese sentido, formuló los primeros enunciados de lo que después se conoció en Colombia como el "Estado social de derecho" ${ }^{19}$.

En relación con los aspectos económicos, la reforma estuvo en parte influenciada por la política intervencionista del "New Deal", promovida para la época por el presidente Franklin Delano Roosevelt en Estados U nidos $^{20}$. De esta forma, se establece como norma constitucional el intervencionismo de Estado en la economía, con el objeto no sólo de racionalizarla, sino también de propiciar una mayor protección del trabajador $^{21}$. Específicamente, el gobierno "buscaba la posibilidad de dotarse de herramientas jurídicas para promover y conducir el desarrollo de la economía nacional, impulsando la industrialización de sectores específicos y fomentando así la consolidación de las relaciones capitalistas modernas" 22 .

La nueva concepción del Estado intervencionistainiciada por L ópez perduró por mucho tiempo en Colombia, inclusive en medio de posteriores y enormes cambios políticos y sociales ${ }^{23}$. Esto indica la importancia de las nuevas realidades a las cuales $L$ ópez quiso dar respuesta mediante sus reformas. Sólo hasta mediados de la década de los setenta, cuando ya comenzaban a asomar en Colombia los vientos del "neoliberalismo", vino a plantearse la necesidad de un cambio en los procesos de consolidación estatal implementados desde 1936.

La reforma constitucional también estableció una nueva forma de concebir la propiedad privada, concepción novedosa para la época en el caso colombiano. La propiedad es concebida ahora a partir de la función social que ésta pueda tener en el sistema económico ${ }^{24}$. El desarrollo de este nuevo principio constitucional se dio a través de la reforma agraria analizada a continuación.

\subsection{L a reforma agraria}

En 1936 el gobierno de L ópez consiguió aprobar en el congreso nacional la reforma agraria, mediante la $L$ ey 200 de 1936 o L ey de Tierras. Esta reforma, la

$\overline{18}$ BOTERO, Sandra, (2006), "La reforma constitucional de 1936, el Estado y las políticas sociales en Colombia", en Anuario Colombiano de Historia Social y de la Cultura, N.o 33, 85-109, Bogotá.

19 El Estado social de derecho aparece en Colombia formalmente en la nueva Constitución Política de 1991, la cual reemplaza la Constitución Nacional de 1886

20 TIRADO, Álvaro (1985), La revolución en marcha y la reforma constitucional de 1936, Universidad Externado de Colombia, 20, Bogotá.

21 CONGRESO DE COLOMBIA, (1936), Acto legislativo N.o 1, reformatorio de la Constitución de 1886, Bogotá, agosto, artículo 11.

22 BOTERO, Sandra, (2006), Op. cit., 91.

23 OCAMPO, José Antonio, (1996), "Crisis mundial y cambio estructural (1929-1945)", en Ocampo, J. A. (editor), Historia económica de Colombia, tercera edición, Tercer Mundo editores, 113, Bogotá.

24 GIRALDO, César (1994), Op. cit., 23. 
primera de este tipo llevada a cabo en la historia del país, incluyó normas sobre la explotación de la tierra, los derechos de los arrendatarios y colonos sobre las tierras de los patronos, así como el mejoramiento de las condiciones laborales para los jornaleros.

La cuestión que pretendía resolver la reforma era encontrar un mecanismo que permitiese romper con el monopolio de la tierra y las relaciones atrasadas de trabajo en el campo, así como establecer formas de indemnización a los grandes propietarios rurales por la pérdi da de sus rentas. El propósito fundamental era entonces transformar el modelo de producción casi colonial en un modelo agrario capitalista, mediante la transformación de la hacienda ${ }^{25}$.

La Ley buscaba acabar con el latifundio y permitir que la tierra se vinculase al proceso productivo, disminuyendo de esta forma el poder político y económico de los terratenientes. Estos grandes propietarios rurales no invertían las rentas en sus propiedades para que éstas se integrasen así a la dinámica del mercado interno y, por el contrario, mantenían el régimen de hacienda que ataba al campesino y no le permitía a éste vincularse a los mercados laboral y monetario.

Como se indicó arriba, esta norma pretendió dar aplicabilidad al principio de la función social de la propiedad establecido en la reforma del 36. L a forma de hacerlo era mediante la posibilidad de otorgar tierras a todos aquellos campesinos que hubiesen trabajado y cultivado territorios que no les pertenecía. Igualmente, prohibió los desalojos de los campesinos que invadían territorios ajenos y facultó al Estado para expropiar tierras en caso de que fuera necesario. Además, decretó la extinción del dominio a los 10 años, es decir, que si una propiedad no estaba debidamente explotada a los 10 años a partir de la expedición de la ley, podía ser expropiada por el Estado y entregada a campesinos sin tierra ${ }^{26}$.

Los propósitos del gobierno con la Ley de Tierras no pudieron ser cumplidos completamente. La razón fue la fuerte reacción en contra de esta norma por parte de la oligarquía latifundista, la cual prácticamente inhibió, mediante distintos mecanismos de presión, la aplicabilidad en la práctica de la reforma. Como respuesta a la misma, los terratenientes crearon un movimiento político denominado APEN (A sociación Patriótica Económica Nacional).

\subsection{L a reforma educativa y el forta- lecimiento de la educación pública universitaria}

Durante la primera administración de López se llevó a cabo una importante reforma en el campo de la educación.

\footnotetext{
25 KALMANÓVITZ, Salomón (2003), Op. cit., 351.

26 Congreso de Colombia, (1936), Ley 200 "Sobre régimen de tierras", diciembre, capítulo, Bogotá.
} 
La reforma fue realizada mediante los cambios implementados en la política educativa entre 1934 y 1938, y también a través de un conjunto de normas legales que legislaron sobre distintos aspectos de la educación. Entre estas normas se destacan, el Decreto-L ey 2214 de 1935, el Decreto-Ley 1917 de 1935, la Ley 32 de 1936, la Ley $2^{\text {a }}$ de 1937, el Decreto-Ley 267 de 1938, la Ley 165 de 1938 y la Ley 91 de $1938^{27}$.

En este período se estableció la educación primaria obligatoria y gratuita para todos los ciudadanos $y$, para este fin, el Gobierno destinó un $10 \%$ del presupuesto nacional a la educación. La reforma concedió, además, la libertad de enseñanza y limitó la intervención de la I glesia en esta actividad. Implantó el control estatal sobre las escuelas secundarias, casi todas en manos de comunidades religiosas y particulares ${ }^{28}$. Este elemento transformó de manera notable las relaciones entre Estado e I glesia caracterizadas hasta ese momento por una profunda injerencia de la misma en varios aspectos de la vida política y social del país, principalmente el educativo. L a consecuencia de este tema de la reforma fue el aumento de la oposición de la Iglesia Católica al gobierno de López Pumarejo.

En relación con la educación superior pública, se llevaron a cabo un conjunto de acciones que impulsaron y fortalecieron la universidad más importante del país, la Universidad $\mathrm{N}$ acional de Colombia. En primer lugar, en 1935 se realizó una reforma universitaria la cual modificó profundamente la estructura jurídica y administrativa de la universidad. La mayor parte de esa estructura continúa vigente en la actualidad.

La reforma promovió la integración de facultades e institutos, la dotación de recursos financieros suficientes, la democratización de sus autoridades (el rector era elegido por un consejo superior, en el que tenían participación profesores y estudiantes), el establecimiento de las libertades académicas y por primera vez, desde su fundación en 1867, de una autonomía rel ativa de la universidad; la participación de profesores y estudiantes en el manejo del claustro, la presencia de la mujer en las aulas de clase, la apertura de nuevas y más diversas carreras, el estímulo y financiamiento a la investigación, los servicios sociales y la función de extensión académica.

Esta reforma permitió llevar a la U niversidad a la vanguardia educativa de la época. Igualmente, el modelo de universidad propuesto en la misma sirvió de estimuló, en años posteriores, a la creación de más universidades públicas en diversas regiones del país.

\footnotetext{
27 HELG, Aline, (2001), (2001), La educación en Colombia 1918-1957: Una historia social, económica y política, Plaza y Janés editores - Universidad Pedagógica Nacional, Bogotá, capítulo III.

28 lbid, 175.
} 
En segundo lugar, el fortalecimiento de la Universidad Nacional se expresó también en la compra de los terrenos y el comienzo de la construcción de la ciudad universitaria de Bogotá. EI propósito era albergar en un solo ambiente a todas las facultades y escuelas que conformaban la universidad, y las cuales se encontraban dispersas por toda la ciudad. Este conjunto de medidas adoptadas durante la primera administración de López Pumarejo constituyeron uno de los impulsos más importantes que ha tenido la U niversidad Nacional durante toda su historia, y marcaron el rumbo futuro de la institución.

Las características de la reforma universitaria se extendieron al resto del sistema educativo, gol peando duramente a la Iglesia, debido a la laicización y liberación de la educación básica y media (secundaria) ${ }^{29}$. Se redujeron las horas semanales de educación religiosa para dar paso a nuevas áreas que hasta ese momento no se habían incluido dentro de los planes de estudio de las escuelas y colegios. Es así como se imparten las primeras clases de educación sexual y las cátedras de filosofía y sociología ${ }^{30}$.

Igualmente, se da importancia por primera vez a las teorías liberales y naturalistas propugnadas por autores antes prohibidos por el clero (Soren Kierkegaard, Friedrich N ietzsche, etc). Se trata de un paulatino proceso de "liberalización del pensamiento" del sistema educativo nacional. Hasta ese momento la educación se encontraba en la práctica enclaustrada en el conservadurismo de la Iglesia Católica quien, para la época, poseía la mayoría de las instituciones educativas existentes en el país.

\subsection{La reforma tributaria}

La reforma tributaria de $1935^{31}$ estableció un muy importante cambio en la normas tributarias que hasta el momento se habían aprobado en Colombia. Mediante la misma se buscaba aumentar el grado de progresividad y equidad de la estructura tributaria hasta el momento vigente; se satisfacía así la vieja aspiración liberal de gravar directamente las rentas de los sectores de mayores ingresos ${ }^{32}$.

En efecto, logró un aumento en los ingresos tributarios percibidos por el Estado a través de mayores impuestos a las grandes empresas e industrias, así como a partir de la elevación del impuesto a la renta. El esquema tributario resultante se caracterizó por una mayor tributación por parte de las rentas derivadas de la posesión de

\footnotetext{
29 ARIAS, Ricardo, (2008), Estado laico y catolicismo integral en Colombia. La reforma religiosa de Alfonso López Pumarejo, Universidad de los Andes, Mímeo, 73, Bogotá 
capital, en relación con las rentas cuya fuente es el trabajo $0^{33}$.

Es así como se aumentó la tarifa del impuesto a la renta para las personas naturales y jurídicas con altos ingresos del 8 al 17\%, se crearon los impuestos al patrimonio y al exceso de utilidades, y se introdujeron cambios en los impuestos sobre la masa global hereditaria, asignaciones y donaciones ${ }^{34}$. No sólo se fortaleció el recaudo, sino que se orientó la estructura tributaria a favor de los impuestos directos, de manera que se relegaron los impuestos de aduanas a un segundo plano $0^{35}$.

El impuesto de exceso de utilidades lo debían pagar quienes obtenían utilidades superiores a una determinada proporción de su patrimonio. En la época fue bastante polémico porque se consideraba como un castigo a la eficiencia. El gobierno lo defendió como un tributo justificado por al aprovechamiento excesivo que hacían ciertas empresas de los beneficios otorgados por la protección aduanera ${ }^{36}$.

La reforma pretendía captar de las franjas más adineradas de la población los recursos necesarios para la realización de obras públicas en el sector de transporte, así como para financiar el gasto público en salud y educación. Esta reforma representó "el único intento en el siglo XX de integrar la acción instrumental con la acción educativa tendiente a aumentar el recaudo con resultados ampliamente positivos" ${ }^{37}$.

Este cambio en la política tributaria del Estado generó enfrentamientos entre el gobierno nacional y los grandes empresarios, acostumbrados hasta ese momento a aportar una cantidad mínima de sus utilidades al fisco.

\section{5 $L$ a reforma laboral}

Durante su primera administración López implementó una política laboral diferente en relación a lo acontecido hasta ese momento en los anteriores gobiernos. Se trató de una de las etapas en donde el Estado estableció un mayor acercamiento con el conjunto de los trabajadores, tanto de la ciudad como del campo.

E se acercamiento se expresó, entre otros aspectos, en el permanente estímulo de parte del gobierno al movimiento sindicalista, lo cual se reflejó en la creación de la Confederación de Trabajadores de Colombia (CTC). En la mayoría de conflictos laborales que tuvieron lugar en esta época el Estado

\footnotetext{
33 LÓPEZ, Héctor, (1998), "Tributación y falta de legitimidad en Colombia", Revista Cuadernos de Economía, Universidad Nacional de Colombia, N.o 28, 157, Bogotá.

34 ALVIAR, Óscar y ROJAS, Fernando, (1985): Elementos de finanzas públicas en Colombia, Editorial Temis, 107, Bogotá.

35 ROBINSON y URRUTIA, Miguel, (2007), Op. cit., 249.

36 GIRALDO, César, (1994), Op. cit, 33.

37 LÓPEZ, Héctor, (1998), Op. cit., 158.
} 
asumió una posición de respaldo a los intereses de los trabajadores, no criminalizando ni reprimiendo la protesta social. Esto hizo que el gobierno de López fuera muy popular entrelas masas de obreros y campesinos.

Durante su primera administración intentó varias veces llevar a cabo una reforma a la legislación laboral hasta ese momento existente. El propósito era reconocer plenamente los derechos y deberes de los trabajadores y consagrar la protección formal del trabajo por parte del Estado.

Sin embargo, la reforma laboral fue, junto con la agraria, una de las que más resistencia tuvo dentro de la élite rural y empresarial del país. Es por esto que dicha reforma sólo pudo ser realizada, pese a todos los inconvenientes políticos y económicos, durante la segunda administración de L ópez Pumarejo entre 1942 y 1945. L os fundamentos de la misma se encuentran en la política laboral aplicada entre 1934 y 1938.

Después del intento de golpe de Estado del 10 de julio de 1944 por parte de un grupo de militares, con el apoyo implícito de la oligarquía terrateniente y parte importante del sector empresarial, el presidente López decretó el estado de excepción o estado de sitio. Una de las medidas que adoptó, aprovechando esa especial situación jurídica, fue la implementación, después de varios años de negativas y rechazo en el congreso, de la reforma laboral.

Esta reforma refleja en parte la situación política de ese momento. EI único sector que apoyó al gobierno durante el intento de golpe fueron los trabajadores organizados en sindicatos y la población en general. El fracaso del mismo se debió en parte importante a la gran movilización popular acontecida durante tres días en respal do al presidente y que al final logró la liberación y restitución en el poder de López Pumarejo.

La reforma laboral finalmente implementada, mediante el Decreto-L ey 2350 de octubre de 1944, desarrolló apartes importantes de la reforma constitucional de 1936, comprendiendo que en una economía en vías de desarrollo, era deber del Estado codificar la relación patrón-obrero ${ }^{38}$. Esta norma fue luego aprobada por el Congreso mediante la L ey 6a de 1945.

Entre los aspectos más importantes se encuentran el reconocimiento del contrato de trabajo como entidad jurídica autónoma39; dio al gobierno facultades para establecer modelos que sirvieran como contrato presuntivo y para fijar el salario mínimo legal vigente; limitación de la duración de la jornada de trabajo a

\footnotetext{
38 OCAMPO, José Antonio, (1996), Op. cit, 122.

39 CONGRESO DE COLOMBIA, (1945), Ley 6” "por la cual se dictan algunas disposiciones sobre la organización del trabajo", febrero, artículo 1.ํ, Bogotá.
} 
ocho horas diarias en la industria y los servicios, a nueve horas en la agricultura40; fijación de una prima del 25 al $50 \%$ por el trabajo nocturno y las horas suplementarias ${ }^{41}$; remuneración del descanso dominical ${ }^{42}$; reconocimiento de indemnizaciones por enfermedades profesionales y accidentes de trabajo e indemnizaciones por enfermedades no profesionales ${ }^{43}$; reglamentación de las "cesantías", equivalentes a un mes de salario por año de trabajo; establecimiento del retiro a los 55 años de edad o tras 20 años de actividad ${ }^{44}$. I gualmente se creó el "fuero sindical" el cual prohibía a los empresarios despedir o licenciar a los dirigentes sindicales.

Esta legislación ha sido considerada como el mayor avance en los derechos social es de los trabajadores en la historia de Colombia ${ }^{45}$. Y su importancia es mayor cuando la gran mayoría de estas prerrogativas fueron luego paulatinamente eliminadas a partir de la década de los noventa del siglo XX, con el advenimiento definitivo del modelo de desarrollo de apertura económica 0 modelo neoliberal. Esta situación se dio, y se presenta en los momentos actuales, bajo la premisa de la "flexibilización laboral".

\subsection{L a reforma constitucional de 1945}

Y a en las postrimerías de su segundo mandato, Alfonso López Pumarejo impulsó una nueva reforma constitucional que fue finalmente aprobada en 1945. Esta reforma pretendía, en general, continuar con el proceso de modernización del Estado colombiano en distintos aspectos, einiciada por el mismo L ópez en 1934.

Son varios los elementos más rel evantes de la reforma. En el aspecto administrativo se destaca la introducción de modificaciones al régimen territorial, más específicamente en relación a los requisitos para la organización de nuevos departamentos y la conformación de Bogotá como distrito especial ${ }^{46}$.

El acto legislativo reformatorio contempla la concesión de la ciudadanía a la mujer pero sin derecho a votar ${ }^{47}$. Sin embargo, esta medida representa un avance importante para la época por cuanto permite a la mujer insertarse formalmente al mercado laboral. EI hecho de poder trabajar, y ser reconocido este trabajo por la ley, aumenta la autonomía económica de la mujer en la sociedad.

40 Ibid, artículo 3..

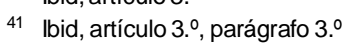

42 Ibid, artículo 7.음

43 lbid, artículo 12.

44 Ibid, artículo 14.

45 MOLINA, Gerardo, (2007), Op. cit., 171.

46 CONGRESO DE COLOMBIA, (1945), Acto legislativo No 1, reformatorio de la constitución de 1886, febrero, artículo 1, Bogotá.

47 lbid, artículo 3. 
La reforma constitucional también establece por primera vez en Colombia desde 1886 la expresa prohibición a los militares de sufragar y de su intervención en política mientras se encuentren en el servicio activo. Este punto es importante porque amplía y consolida el poder civil sobre las fuerzas armadas nacionales y evita su posible influencia en los procesos políticos y electorales ${ }^{48}$.

Otro de los aspectos de la reforma es la ampliación de las responsabilidades del Congreso respecto de la discusión y aprobación de proyectos de orden económico. Más específicamente, se amplían las responsabilidades de discusión en relación con la planeación económica por programas y algunos elementos fiscales como el establecimiento y ampliación de las rentas públicas, el reconocimiento de la deuda del nivel nacional y la creación de empleos públicos ${ }^{49}$.

Uno de los elementos más importantes es respecto de la función económica del Estado. La reforma constitucional de 1945 amplía el campo de acción de la intervención económica del Estado. Éste puede ahora intervenir "en la explotación de industrias o empresas públicas y privadas, con el fin de racionalizar la producción, distribución y consumo de la riqueza" ${ }^{50}$. Esto posibilita la creación de empresas públicas y aparece entonces la figura del Estado como actor principal en el sistema económico.

E ste aspecto complementa lo ya establecido en la reforma de 1936 impulsada por López. Es en ese norma donde aparece por primera vez en la legislación colombiana la intervención del Estado en la economía como una de sus funciones primordiales.

\section{Conclusiones: una breve interpre- tación de las reformas económicas y sociales implementadas por Alfonso López Pumarejo}

Los dos períodos de gobierno de A Ifonso López Pumarejo representaron un intento de "modernizar" las relaciones económicas y sociales existentes en ese momento en Colombia, utilizando como instrumento principal la modernización del Estado y el marco legal y jurídico sobre el cual se respaldaba la actuación de este $\mathrm{E}$ stado.

La "modernización" significa un intento de transformación de las relaciones económicas y sociales propias de una economía subdesarrollada primarioexportadora. En ese sentido, se pretendía "romper" con los esquemas y relaciones "cuasifeudales" y latifundistas que caracterizaban la economía y la sociedad colombiana de la época. Ese tipo de relaciones generaban unas estructuras

\footnotetext{
48 Ibid, artículo 168.

$49 \mathrm{lbid}$, artículo 7.

50 lbid, artículo 4.
} 
económicas atrasadas e ineficientes que impedían una inserción más "creativa" (diversificada) a los mercados internacionales ${ }^{51}$.

En la primera mitad de la década de los treinta del siglo XX existía en Colombia una difícil situación económica y social. Por una parte, se presentaba una crisis en el proceso de acumulación financiado con crédito externo y basado en la exportación de productos primarios. La gran depresión mundial había interrumpido el flujo de capitales desde el exterior y generado una disminución de las exportaciones de productos fundamentales para el comercio exterior como el cafés2. Por otro lado, se asistía a una crisis de legitimidad del sistema político vigente fundamentado en el dominio del partido conservador, de los terratenientes 0 grandes propietarios rurales y la Iglesia C atólica ${ }^{53}$.

A nte este contexto, aparecía entonces la necesidad de un nuevo proceso de acumulación y un replanteamiento del modelo de Estado que respondiese a esa nueva lógica. El proceso de acumulación debía basarse ahora en la expansión del mercado interno ${ }^{54}$. El Estado debía ser reformado para que creara las condiciones económicas y sociales necesarias para viabilizar estas transformaciones.
El intento de "modernización" de las dos administraciones de Alfonso López $P$. se expresa en el conjunto de reformas propuestas y realizadas entre 1934 a 1938 y 1942 a 1945. El componente principal de las mismas es el cambio en el concepto de Estado vigente hasta ese momento en Colombia. El Estado era concebido como un simple árbitro que no tenía mayor injerencia en la economía y la sociedad. La transformación se dirige hacia un concepto de Estado más dinámico, con una mayor capacidad de intervención en la economía y en la solución de los conflictos sociales.

Es por esto que el eje central de las transformaciones propuestas se concentró en la reforma constitucional de 1936. Esta reforma constituyó el cambio más importante realizado a la Constitución Nacional de 1886 hasta ese momento, y a la postre, uno de los dos cambios más trascendentales efectuados durante la vigencia de esa carta constitucional (entre 1886 y 1991) ${ }^{55}$. La reforma de 1936 constituyó el eje central porque en ella se establecieron una serie de principios generales sobre el Estado, la economía, la educación, la propiedad y las relaciones laborales, que luego serían intentados desarrollar a través de reformas específicas para cada tema.

\footnotetext{
51 La intencionalidad de "modernización" y transformación pretendía adaptar la economía y la sociedad colombiana a los cambios que ya comenzaban a expresarse en el conjunto del capitalismo mundial sin romper con ese mismo capitalismo. 52 GIRALDO, César (1994), Op. cit., 13.

53 Ibid, p.13.

54 lbid, p.13.

55 La otra gran reforma de importancia realizada a la Constitución Nacional de 1886 se realizó en 1968 por parte del presidente Carlos Lleras Restrepo.
}

\section{8}


L as características del nuevo Estado serían en lo fundamental, la intervención económica, la defensa de la industria y la agricultura frente a la competencia externa a través de políticas proteccionistas, el fomento del crecimiento industrial mediante la canalización de recursos internos, la introducción de reformas sociales y en el sistema de tenencia de la tierra. Se trataba de otorgarle a ese Estado una mayor autonomía frente a los terratenientes $y$, en general, frente a los distintos grupos de presión, para poder así absorber y canalizar institucionalmente los conflictos sociales y también para dar salida a algunas de las aspiraciones de las capas medias ${ }^{56}$.

Este tipo de Estado representaba en lo fundamental los intereses de una nueva clase empresarial nacional que propendía por la industrialización del país. Para ello se requería de un nuevo marco institucional que regulase las relaciones sociales y económicas con el objeto de garantizar la paz social. También requería remover las formas tradicionales de dominio en las zonas rurales para así permitir el desarrollo de las fuerzas productivas del campo. Con este desarrollo se ampliaría la capacidad de consumo de la población, se aumentaría la oferta de productos agropecuarios a la industria y se liberaría la circulación de la mano de obra asalariada ${ }^{57}$.

\footnotetext{
56 KALMANÓVITZ, Salomón, (2003), Op. cit., 365.

57 LÓPEZ PUMAREJO, Alfonso, (1979), Op. cit. p.25.
}

El discurso político de Alfonso López Pumarejo, si bien servía a los intereses de industriales y cafeteros, era más universal e involucraba transformaciones más profundas. Por tal razón, estas clases inicialmente le abrieron el paso, pero cuando quiso ir más allá para lograr el cumplimiento cabal de su programa de gobierno, estos mismos sectores comenzaron un proceso de oposición hacia el avance de las reformas.

El intento de modernización y transformación enfrentó entonces la tenaz resistencia de parte importante de la élite económica y política. Los grandes propietarios de la tierra, poseedores de extensos e improductivos latifundios, fueron los principales opositores a las reformas. Estos se encontraban representados en instituciones como la I glesia, el Partido Conservador y un sector del mismo partido de gobierno, el Partido Liberal.

El alcance de las transformaciones propuestas fue limitado principalmente por las resistencias de los sectores sociales mencionados. Es por esto que es más factible hablar de "intencionalidad" de cambio, de mudanza, que de consecuencias profundas en la estructura económica durante los dos gobiernos de A Ifonso López Pumarejo. 
Un ejemplo de la importancia de estas resistencias se encuentra en el intento de golpe de Estado que tuvo lugar en 1944, durante la segunda administración (1942-1945). A la postre, fue esta oposición sistemática de la oligarquía latifundista y otros sectores, entre otros factores, la que detuvo el proceso de transformaciones propuesto, y que conllevaron a la renuncia de López en 1945, a un año de la finalización formal de su segundo período presidencial. Parte de las consecuencias de no completar el proceso de modernización capitalista del Estado, la economía y la sociedad propuesto por López Pumarejo, explican de alguna forma las condiciones actuales de subdesarrollo, pobreza, violencia y gran debilidad del Estado frente a los intereses de las élites económicas y políticas que caracterizan hoy a Colombia.

\section{Bibliografía}

ALVIAR, Óscar y ROJAS, Fernando, (1985): Elementos de finanzas públicas en Colombia, Editorial Temis, 107, Bogotá.

ARIAS, Ricardo, (2008), Estado laico y catolicismo integral en Colombia. La reforma religiosa de $\mathrm{A}$ Ifonso López Pumarejo, Universidad de los A ndes, Mímeo, 73, Bogotá.

BOTERO, Sandra, (2006), "La reforma constitucional de 1936, el Estado y las políticas sociales en Colombia", en A nuario Colombiano de Historia Social y de la Cultura, N. 33, 85-109, Bogotá.

CONGRESO DE COLOMBIA, (1936), A cto legislativo $N .^{\circ} 1$, reformatorio de la Constitución de 1886, Bogotá, agosto, artículo 11.

Congreso de Colombia, (1936), Ley 200 "Sobre régimen de tierras", diciembre, capítulo, Bogotá.
CONGRESO DE COLOMBIA，(1945), A cto legislativo No 1, reformatorio de la constitución de 1886, febrero, artículo 1, Bogotá.

CONGRESO DE COLOM BIA, (1945), Ley 6a "por la cual se dictan algunas disposiciones sobre la organización del trabajo", febrero, artículo 1.ํ, Bogotá.

GIRALD0, César, (1994), Estado y hacienda pública en Colombia 1934-1990, Contraloría General de la República, 14-15, Bogotá.

HEL G, A line, (2001), (2001), La educación en Colombia 1918-1957: Una historia social, económica y política, Plaza y J anés editores - U niversidad Pedagógica N acional, Bogotá, capítulo III.

K A L M A N ÓVITZ, Salomón, (2003), Economía y nación: U na breve historia de Colombia, 2. a edición, Editorial Norma, 366, Bogotá. 
LÓPEZ PUMAREJ0, A Ifonso, (1979), Obras selectas, dos(2) volúmenes, compilación de Jorge M ario Eastman, Imprenta Nacional - Cámara de Representantes de Colombia, 20, Bogotá.

LÓPEZ, Héctor, (1998), "Tributación y falta de legitimidad en Colombia", Revista Cuadernos de Economía, Universidad Nacional de Colombia, N.28, 157, Bogotá.

MELO, Jorge Orlando, (1991), "Las reformas liberales de 1936 y 1968. Progreso social y reorganización del Estado", en Revista Credencial Historia, N. ${ }^{\circ} 13,1$, Bogotá.

MOLINA, Gerardo, (2007), Las ideas liberales en Colombia: 1849-1959, 5. a edición, U niversidad Libre, 20, Bogotá.

OCAMPO, José Antonio y M ONTENEGRO, Santiago, (2007), Crisis mundial, protección e industrialización. Editorial Norma, 2. a edición, 20, Bogotá.

OCAM PO, José A ntonio, (1996), "Crisis mundial y cambio estructural (1929-1945)", en Ocampo, J. A .

(editor), Historia económica de Colombia, tercera edición, Tercer M undo editores, 113, Bogotá.
ROBINSON, James y URRUTIA, M iguel, (2007), Economía colombiana del siglo XX. Un análisis cuantitativo, Fondo de Cultura Económica - Banco de la República, 393, Bogotá.

SANTOS, Enrique, (2005), "Las revolucionaria República Liberal", en Revista Credencial Historia, N. $.183,1$, Bogotá.

TIRA D O, Álvaro (1985), La revolución en marcha y la reforma constitucional de 1936, Universidad Externado de Colombia, 20, Bogotá.

TIRADO, Álvaro (compilador), (1986), Estado y economía: 50 años de la reforma del 36, Contral oría General de la R epública, 16, Bogotá.

TIRADO, Álvaro, (1995), A spectos políticos del primer gobierno de Alfonso López Pumarejo, Editorial Planeta, 35, Bogotá.

URREG0, Miguel, (2005), La revolución en marcha en Colombia (1934-1938): U na lectura en perspectiva latinoamericana, U niversidad M ichoacana de San N icolás de Hidalgo - Instituto de Investigaciones Históricas, 65, M ichoacán, M éxico. 